\title{
Preferencias y disposición a pagar de la sociedad por programas de inclusión social: el caso español
}

\section{Matías M. González Hernández Yen E. Lam González}

RESUMEN: Esta investigación tiene como objetivo analizar las preferencias de la sociedad por programas de inclusión social. Como resultado de encuestar a una muestra de 1250 residentes en Canarias (España) se ha encontrado que existe una alta sensibilidad al precio de los programas. Además, la sociedad muestra una alta preferencia por la renta básica, con una DAP superior a 60 euros, mientras que los programas de sensibilización son los menos valorados con una DAP inferior a 38 euros anuales. El estudio permite formular un paquete de orientaciones en materia de política social, para elevar la eficacia de los programas de incentivos y sensibilización, tanto para administraciones públicas como para el conjunto de factores que contribuyen a esta dimensión del desarrollo.

PALABRAS CLAVE: Exclusión Social, Política Social, Desarrollo, Economía Social-Solidaria, Pobreza.

CLAVES ECONLIT: A130, D120, D630, I31.

Cómo citar este artículo/How to cite this article: GONZÁLEZ HERNÁNDEZ, M. \& LAM GONZÁLEZ, Y.E. (2021): "Preferencias y disposición a pagar de la sociedad por programas de inclusión social: El caso español”, CIRIEC-España, Revista de Economía Pública, Social y Cooperativa, 103, 225-248. DOI: 10.7203/CIRIEC-E.103.18283.

Correspondencia: Matías M. González Hernández, Universidad de Las Palmas de Gran Canaria (ULPGC), Facultad de Economía, Empresa y Turismo, Doctor en Economía, matiasmanuel.gonzalez@ulpgc.es; Yen E. Lam González, Universidad de Las Palmas de Gran Canaria (ULPGC), Facultad de Economía, Empresa y Turismo, Doctor en Economía, yen.lam@ulpgc.es 
ABSTRACT: This paper aims to assess public preferences and WTP for policies addressing social inclusion. As a result of surveying 1250 residents in the Canary Islands (Spain) it has been found that society is highly sensitive to price, since people always choose to pay the cheapest option in support of social inclusion. In addition, the interviewees show a greater preference for basic income as a form of policy with a WTP of about 60 euros, while the WTP for other programmes related to awareness are less than 39 euros/year. The study allows to formulate a package of recommendations to increase the effectiveness of current policies aiming to promote a more inclusive and resilient society.

KEYWORDS: Social exclusion, policy, development, social economy, solidarity, poverty.

\section{Expanded abstract}

\section{Public preferences and willingness to pay for social inclusion programmes: The Spanish case}

Poverty and social exclusion are characterised as deprivation and lack of opportunities to lead each person's life worth living. Nevertheless, social exclusion is not only a concern of public policies but also of society. Individuals have practical tools to fight against social exclusion since they make their choices regarding those public and social programs they want to support (Littlewood \& Herkommer, 2017; Martín, Formichella, \& Costabel, 2020).

In the Canary Islands (Spain) over the last five years, the evolution of the most relevant economic indicators leaves no doubt about the dramatic unemployment scenario and the increase of the social contingent in a situation of poverty and social exclusion. Long-term unemployment is considered the primary vehicle through which more and more people slide towards situations of poverty and exclusion. This situation will undoubtedly be worsened by the global health emergency caused by COVID 19, which will exponentially affect social inequality in Spain. The complete inactivity of tourism in the Canary Islands region, a sector representing about $30 \%$ of the economic structure and more than $40 \%$ of the jobs in the archipelago, are among the leading causes of the sharp deterioration in the employment figures (Palomino et al., 2021).

At the same time, the Canary Islands have a wide range of organizations and actors working for social inclusion, and in general, to establish a new framework of economic relations based on solidarity values. This diversity of initiatives and actors positions the archipelago as an ideal scenario for analysing and experimentation the social preferences for public policies (González Hernández et al., 2013). 
This paper aims to assess the preferences of society and willingness to pay (WTP) for several programs addressing social inclusion. Four main programs were analysed, and several improvements for each program were proposed as policy alternatives. The four programs were:

i) to provide a basic income to vulnerable groups,

ii) to provide support and funds to occupational insertion companies or solidarity organizations,

iii) to implement a responsible public purchasing program, and

iv) to increase sensibilization programs targeting the society to promote a more responsible consumption that supports social inclusion initiatives.

The application was based in the Canary Islands (Spain), where 1250 residents were interviewed using a structured questionnaire. Individuals were posed with hypothetical scenarios in which various combinations of policy interventions were in place at a given price. Policy combinations were randomly formed by running the statistical program Ngene with fifteen policy alternatives levels of the four main social inclusion programs mentioned above.

Each individual answered to four choice cards, with each card being a combination of two policy scenarios versus a third option which is the current situation. Each hypothetical scenario of policy alternatives was assigned with a price, which represented an annual fee that all citizens would have to pay in case of implementing such measures. The "status quo" scenario had a price of $0 €$, representing the current situation concerning ongoing subsidy programs and support to social exclusion groups and companies that employ vulnerable groups. Other aspects related to the socio-demographic characteristics of respondents, their level of knowledge about social inclusion programs and sensitiveness with the problem were also included in the questionnaire.

As a result, it has been found that individuals are susceptible to price since respondents always chose to pay the cheapest program in support of social inclusion. Interviewees show a greater preference for basic income as a form of policy. In other words, subjects are willing to pay higher amounts for those programs that pay directly to people at risk of poverty or exclusion (PROGRAM $1=€ 60.30$ ) than for policies that promote the consumption of goods and services provided by solidarity organizations, which are dedicated to hiring people in this type of situation (PROGRAM $4=€ 38.00$ ). At the socio-demographic level, data indicate that single men are more likely to pay more for social inclusion. Likewise, as expected, having a job or an own economic activity also increases the probability of choosing the combination of policies with the highest price.

Results suggest that the Canary Islands must make an even greater effort to make society more sensitive about poverty and social exclusion programs, on the one hand, and to make more informed decisions based on efficiency enhancement criteria when choosing a social inclusion program on the other hand. This requires reorienting policies towards a decrease of subsidies and stimulating demand and social awareness.

In the context of economies with a strong specialization in tourism, such as the Canary Islands, recommendations can be extended to tourists by promoting the consumption of local products and services that generate a positive, mutually beneficial link between tourism 
companies and solidarity economy entities, which is desirable from the point of view of the sustainability of both.

This study faces various limitations that substantially reduce the potential generalization of results and the scope of its conclusions to the Spanish case study. First, it is based on a single Autonomous Community, "The Canary Islands". Second, further evidence with much larger samples is required to prove the robustness of the model definitively. Third, this research has focused on the current situation of the archipelago concerning social inclusion programs and a proposal of viable scenarios, from redistributing public funds to new inclusion policies. However, the extrapolation of results for other policy contexts requires further knowledge of the other sub-national contexts, and future studies should consider including a larger sample of citizens and Spanish regions. Finally, there might also be a need to include the importance-performance evaluation of citizens in the studied programs. Nevertheless, this study can be seen as a methodological guide of potential application to the Spanish case. 


\section{Introducción}

La pobreza y la exclusión social son generalmente caracterizadas como estados carenciales y de ausencia de oportunidades para llevar a cabo la vida que cada cual considera que merece la pena vivir, bien por la privación de oportunidades, de ausencia de vínculos sociales o familiares para la atención de necesidades materiales e inmateriales, situaciones permanentes de baja 0 nula empleabilidad debido a bajas capacidades, entre otros (Agulnik, 2002; Littlewood \& Herkommer, 2017; Schiller, 1989). Asimismo, la exclusión social tiene un carácter multidimensional como conductora de los procesos de vulnerabilidad y exclusión, abarcando diferentes aspectos del desarrollo humano (Martín, Formichella, \& Costabel, 2020; Navarro \& Arce, 2016).

Por su parte, la práctica ha demostrado que la exclusión social no es solamente una preocupación de las políticas públicas y de grupos organizacionales, sino también de la propia sociedad, pues posee herramientas de lucha y apoyo efectivas, ya que cuanto mayor sea la financiación a los programas públicos y sociales (que también provienen de la sociedad), más personas podrán ser atendidas y beneficiadas (Casassas, 2013).

En Canarias (España) a lo largo del último lustro, la evolución de los indicadores económicos más relevantes no deja lugar a dudas sobre el dramático escenario de desempleo, y el aumento del contingente social en situación de pobreza y exclusión social, siendo el desempleo de larga duración, principal vehículo por el que se deslizan importantes contingentes sociales hacia situaciones pobreza y exclusión, el indicador de mayor crecimiento (EAPN-España 2017; González Hernández et al., 2013). Dicha situación sin dudas se verá empeorada a causa de la crisis sanitaria global de 2020 (COVID 19), que tendrá un efecto exponencial en la situación de desigualdad social en la que se encuentra el archipiélago español (Palomino et al., 2021), lo que debe su causa fundamental a la completa inactividad turística en la región, sector que representa un 30\% de la estructura económica y más del $40 \%$ de los empleos en el archipiélago.

A la vez, Canarias exhibe un amplio crisol de organizaciones y actores que trabajan por la inclusión social de grupos sociales excluidos o en riesgo de estarlo, y en general, por el establecimiento de un nuevo marco de relaciones económicas sustentado en valores solidarios. Esta diversidad de iniciativas y actores hacen que la situación del Archipiélago canario sea extraordinariamente compleja, y se muestre como un interesante laboratorio de análisis y experimentación del estudio de este fenómeno sistémico, la evaluación de las políticas públicas implementadas en este ámbito, y más importante, la percepción y valoración de la sociedad, que es la que se encuentra más cercana a este núcleo de personas, y tienen la opción de apoyar, comunicar y actuar en favor de estos grupos, a través de las organizaciones, mediante el trabajo voluntario, de forma directa, y de una manera indirecta, a través del consumo de productos y servicios provenientes de las entidades que luchan por la integración social (González Hernández et al., 2013).

Así, este estudio se plantea como objetivo fundamental evaluar las preferencias y la disposición a pagar (DAP) de la sociedad canaria por diversas medias que persiguen activa y conscientemente mitigar la exclusión social en esta región del Atlántico. Para ello se han planteado varios experimentos de elección discreta y un modelo de regresión logística, para generar una 
base de conocimiento empírico que pueda ser estudiado en otros contextos. También se analiza el efecto de variables a nivel socio-demográfico de los individuos, el nivel de renta y la satisfacción en su DAP. Otros aspectos relacionados con el grado de conocimiento que tiene la sociedad canaria sobre las diversas situaciones que conllevan a la exclusión social, y las causas que la originan también son analizados. El estudio ha sido financiado por la Unión Europea a través del Programa de Cooperación Transnacional Açores-Madeira-Canarias (MAC) y del proyecto "ECOS" "Sustentabilidad Integrada de las Empresas y Organizaciones de Economía Solidaria en la Macaronesia (2007-2017).

\section{Marco teórico conceptual}

Aun cuando existen diversos enfoques y criterios relativos al término pobreza, hay consenso respecto a que es un término que viene directamente ligado a niveles bajos de ingresos económicos (Decerf, 2021; Nolan \& Whelan, 1996; Schiller, 1989). Un ejemplo de ello es la definición de pobreza relativa, la que se mide hoy como aquel nivel de ingresos inferior a la mitad o menos de la media de un grupo poblacional. Al margen de esto, se han ido elaborando otras formas de aproximación al fenómeno de la pobreza, con índices de pobreza que no sólo tienen en cuenta los ingresos, sino también el gasto, el consumo, la educación, etc., lo que también varía en función del contexto analizado, el país o la sociedad (Decerf, 2021).

Por otro lado, el crecimiento de otras fuentes de desigualdad social tan o más importantes que la renta, como por ejemplo la irregularidad administrativa que sufren un gran número de extranjeros sin papeles, la difícil inserción social de un ex recluso o un drogodependiente entre otros (Gottlieb, 2017), o incluso tener hijos (AROPE; 2020) da pie a otros escenarios de análisis. El concepto de exclusión social se revela entonces como extraordinariamente útil para hablar de todas aquellas situaciones en que, más allá de la privación económica, se sufre de una privación de oportunidades, de escasez de redes sociales o familiares, dificultades de acceso al mercado formal de trabajo, la baja formación, la mala salud, etc., las que a su vez se acumulan en situaciones de carencia económica (Agulnik, 2002; Littlewood \& Herkommer, 2017).

Así como la pobreza se ha asociado a la falta de recursos económicos, la exclusión social no se puede explicar con arreglo a una única causa o factor, sino que precisamente se define por una acumulación de factores o déficits que se interrelacionan y retroalimentan entre sí. La exclusión social ha sido considerada, desde hace algunos años, como un proceso de creciente vulnerabilidad que afecta a sectores cada vez más amplios del cuerpo social, y tiene un fuerte impacto emocional y socio-psicológico en las personas (Sjåstad et al., 2021). Hoy se materializa en la carencia creciente de oportunidades a nivel laboral, residencial, económico. En la realidad actual, el universo social expuesto a este tipo de situaciones, es cada vez más amplio; madres monoparentales, ex reclusos, inmigrantes en situación irregular, y personas en situaciones de desempleo por más de un año, son solo algunos ejemplos de ello (Felber, 2012; Littlewood \& Herkommer, 2017). 
Para apoyar a estos colectivos a salir de la situación de pobreza y exclusión, pueden llevarse a cabo diversos programas públicos. El éxito de estos programas dependerá de la financiación que obtengan del Estado y la sociedad y obviamente cuanto mayor sea la financiación de esos programas, más personas podrán ser atendidas y beneficiadas (Pereznuñez, 2005). A grandes rasgos estos programas pueden clasificarse en tres grupos fundamentales:

- Renta básica y servicios gratuitos a familias con personas en pobreza y exclusión, para cubrir sus necesidades fundamentales (alimentos, vivienda, educación, etc.).

- Subvencionar a las empresas que contratan y dan formación a personas en situación de pobreza o exclusión social (empresas de inserción, centros de empleo, empresas de economía social y solidaria, etc.).

- Informar y sensibilizar a la sociedad y las propias administraciones públicas para que se implique en apoyar a las personas pobres y excluidas, mediante el consumo de la Economía Solidaria y el trabajo voluntario en empresas de inserción.

En la actualidad, se suma el crecimiento y desarrollo de diferentes movimientos o grupos organizacionales, que bajo diferentes etiquetas, se han implicado en la promoción práctica de modelos de desenvolvimiento de las economías centradas en los seres humanos y la utilidad social (Castro Núñez et al., 2013), equitativos, democráticos, y ambientalmente viables (Lee, 2020). Aquí se encuentra la denominada economía social y/o solidaria (en lo adelante ES), compuestas por un amplio y heterogéneo conjunto de actores económicos (en lo adelante EES) que no solo emplean y forman a personas en situación de riesgo, sino que difunden valores e información, incidiendo en la visión, actitudes y comportamientos del conjunto de los demás actores económicos fundamentales y pretendiendo lo mismo en la sociedad (Castro Nuñez et al., 2013; Delgado \& Richer, 2001). Los estudios confirman que la las EES contribuyen a generar y preservar empleo en los sectores económicos tradicionales y emergentes, y promueven el trabajo estable con un sólido enfoque de género e inclusivo (Borzaga et al., 2019).

En resumen, dentro de este sector de empresas se encuentran ; i) las entidades enfocadas en el trabajo de inserción laboral, ii) las organizaciones de consumo y/o la producción responsable, y sus actores representantes, que realizan, o al menos intentan, decisiones de consumo que favorezcan modelos de producción amables con el entorno, respetuosos con las culturas locales y basadas en relaciones económicas basadas en la gestión participativa y la democracia económica (por lo general se agrupan como asociaciones y/o fundaciones), iii) los actores dedicados a innovar en el sistema financiero (banca ética), basados en principios de democracia económica, de medios financieros accesibles a grupos en riesgo de exclusión. Por último, otros grupos de entidades que bien actúan en aras de favorecer a personas que se encuentran en situación de exclusión o en riesgo de estarlo, colaborando en su integración a la sociedad, o bien que difunden los valores pro-inclusión social, comercio justo, finanzas éticas y economía social y solidaria en general (Horrach \& Salvà, 2011; Salvà \& Rosselló, 2013; Torresano et al., 2015).

Es importante destacar que muchas EES pueden presenciar o abordar en su quehacer diversos ámbitos, bien como oferentes o como demandantes. Es muy común, en este sector, que todas las entidades participen y organicen acciones de sensibilización y difusión, así como también que opten por convertirse o asociarse a grupos de consumidores responsables. La 
inserción laboral por su parte, es otro ámbito de actuación que no solo se ciñe a las empresas que se dedican a la inserción laboral. La formación, es una actividad muy periódica y común a la vez para la totalidad del sector (Horrach \& Salvà, 2011; Torresano et al., 2015).

La creación y desarrollo de nuevos modelos y herramientas de gestión, de acuerdo a los principios de la ES, que no se limitan a copiar y / o adaptar los que fueron y son producidos por/para el sector empresarial de la economía, particularmente en las áreas en las que es esencial proponer y practicar la diferencia: Gestión de Personas y sus comportamientos, Incentivos y Talento (mejor que "Gestión de Recursos Humanos"), gestión de las relaciones con proveedores y clientes (en la línea de "Gestión de Empresas"), Sistemas de Comunicación y Visibilidad (en lugar de "mercadeo social"), sistemas de monitoreo y evaluación integradas (no sólo el tradicional de contabilidad económica y financiera, sino también teniendo en cuenta las otras dimensiones (social, cultural, ambiental territorial, político y cognitivo del tamaño de la administración).

Sea cual sea la visión que se tenga de la economía solidaria, el hecho cierto es que, en palabras de André Mance (2007), millones de personas practican la economía solidaria a lo largo y ancho del mundo, trabajando y consumiendo con la finalidad de obtener bienes para su propio bienestar y el de otras personas, en lugar de orientarse por la obtención del lucro. En una ES lo que importa es la creación de unas condiciones económicas satisfactorias para toda la gente, lo que implica asegurar una libertad personal y colectiva, además de abolir toda forma de explotación, dominación y exclusión, protegiendo los ecosistemas y promoviendo un desarrollo sostenible (Castro Nuñez et al., 2013).

Y por último, la sociedad, que en este sentido también se convierte en un eje fundamental de apoyo a la ES, ya que es la que se encuentra más cercana al núcleo de personas en situación de exclusión social o desempleo, a través de las redes sociales, familia, amigos etc., y que en un sentido más estricto tienen la opción de apoyar, comunicar y actuar en favor de estos grupos, a través de las organizaciones, mediante el trabajo voluntario, de forma directa, y de una manera indirecta, a través del consumo de productos y servicios provenientes de las entidades que luchan por la integración social. Así, la exclusión social no es solamente una preocupación de orden público y organizacional, sino también de la propia sociedad, pues posee las mejores herramientas de lucha y apoyo a los programas públicos y las iniciativas privadas (Pedreño, 2010). 
Cuadro 1. Tipología de actores que intervienen en la lucha contra la pobreza y la exclusión social

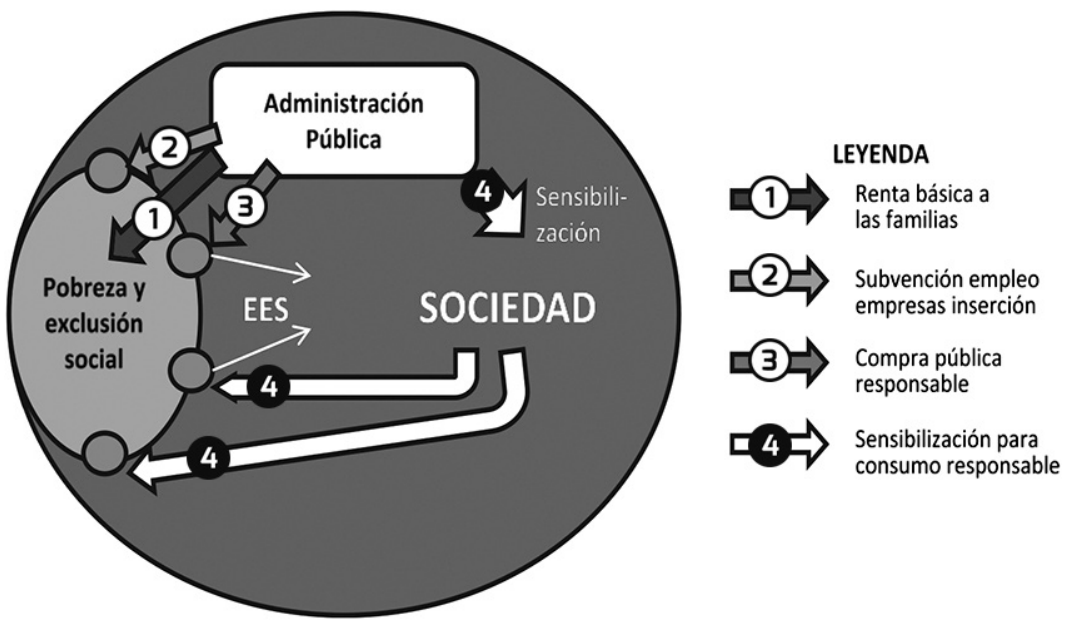

Fuente: Elaboración propia

\section{Metodología}

En esta sección se presenta el área de estudio y el proceso de construcción del modelo y el cuestionario, así como la medición de las variables que se utilizaron en el análisis de los datos.

\section{1. Área de estudio}

El área de estudio es la Comunidad Autónoma de Canarias (España). Las tasas alarmantes de desempleo por las que atraviesa esta CCAA no parecen mejorar desde el impacto de la crisis económica nacional (Bono, 2012). La región alcanzó su máximo histórico de desempleo en 2013 con un total de 295.824 personas. En 2020 antes de la crisis sanitaria causada por COVID, la CCAA aún no lograba bajar de la cifra de los 200 mil (Hodgson Garabote, 2019).

Con estas cifras, Canarias junto con Andalucía, son las únicas dos Comunidades Autónomas (CCAA) españolas que presentan una tasa de desempleo de nacionalidad española superior a la extranjera, según los datos del primer trimestre de 2019 procedentes de la Encuesta de Población Activa, EPA. Según esta fuente, ambas CCAA tienen una tasa de paro para los trabajadores españoles del $27 \%$. 
La situación social en la región es alarmante, pues el 18,4\% de la población no puede llegar a fin de mes. Se estima que solo unas 10.500 personas son beneficiarias de la Prestación Canaria de Inserción (una renta de unos $500 € /$ mes) y unas 1.600 personas tienen contrato subvencionado en empresas de inserción, que significa alrededor de un $4 \%$ del total de desempleados (González Hernández et al., 2013). Este panorama sin dudas ha provocado un incremento del riesgo de pobreza y exclusión social en las islas, que ostentan la segunda tasa más alta de España, con un 38,2 \% de la población (EAPN-España 2018; Llano Ortiz 2016).

Asimismo, las plataformas de lucha contra los problemas de exclusión social y pobreza se han vuelto flexibles al calor de las nuevas manifestaciones sociales. Los actores vinculados a la economía social-solidaria tienen una estructura heterogénea y organizativamente dispersa, y están presentes a lo largo de todo el ciclo de la actividad económica, desde la producción de materias primas e insumos, la financiación, la producción y el consumo de bienes y servicios y hasta el tratamiento de los desechos al final de la vida útil de éstos. El desarrollo experimentado por los movimientos de Economía Solidaria en Canarias, aunque es significativo, es aún incipiente y su alcance es muy limitado en relación con la dimensión de los problemas de exclusión social que se presencian. Fuera necesario expandir el sector, cien veces más, para revertir los actuales problemas de exclusión social (González Hernández et al., 2013).

Aunque evidentemente, la exclusión social posee infinitos vasos comunicantes con el conjunto del cuerpo social y de los fenómenos que lo modelan, la sociedad canaria tiene un papel primordial. De contar con la información suficiente para valorar y sensibilizarse respecto a los problemas que le rodean, mejoraría sus actitudes y disposición respecto a apoyar a estos grupos, lo que contribuiría a que ganaran cada vez mayor peso en los programas de financiación de las Administraciones Públicas, especialmente los Centros Especiales de Empleo (CEE) y a las Empresas de Inserción (EI), ejes vertebradores del futuro sostenible de la Economía Social y Solidaria en el archipiélago (González Hernández et al., 2013).

\subsection{El modelo}

El propósito fundamental del estudio es evaluar las preferencias de la sociedad canaria por diversos programas que persiguen activa y conscientemente mitigar la exclusión social. Para ello se propone un modelo de elección discreta con preguntas relativas a la valoración económica de dichas políticas en función de su contenido solidario, medidas a través de la disposición a pagar de la sociedad por servicios a personas en exclusión o riesgo de estarlo.

Más específicamente se diseña un modelo ASC logit que incluye cuatro alternativas de programas de inclusión. El modelo plantea que la probabilidad de elegir una combinación de políticas y precio depende de una serie de variables, que para este caso se han definido en i) _sexo ii),_estado civil iii)_formación iv)_ocupación v)_nivel de ingresos vii)_situación económica y viii)_satisfacción (ver tabla 1). 


\section{Tabla 1. Variables incluidas en el modelo}

\begin{tabular}{ll} 
Variable & Descripción \\
\hline PROGRAMA & $\begin{array}{l}\text { Variable categórica que toma valor } 1 \mathrm{si} \text { el individuo elige una } \\
\text { alternativa que contiene la política, y } 0 \text { si lo contrario }\end{array}$ \\
\hline _sexo & Variable dicotómica que toma valor 1 si hombre y 0 si mujer \\
\hline _estado civil & $\begin{array}{l}\text { Variable dicotómica que toma valor } 1 \text { si el individuo no tiene } \\
\text { pareja y } 0 \text { si lo contrario }\end{array}$ \\
\hline _ormación & $\begin{array}{l}\text { Variable nominal de cuatro categorías que indica el nivel } \\
\text { educacional del individuo }\end{array}$ \\
\hline _ocupación & $\begin{array}{l}\text { Variable nominal de cuatro categorías que indica la situación } \\
\text { laboral del individuo }\end{array}$ \\
\hline _ivel de ingresos & $\begin{array}{l}\text { Variable de escala con cuatro intervalos de ingresos a nivel } \\
\text { personal del encuestado }\end{array}$ \\
\hline _satisfacción & $\begin{array}{l}\text { Indica el nivel adquisitivo, según la propia declaración del } \\
\text { individuo }\end{array}$ \\
\hline
\end{tabular}

Fuente: Elaboración propia.

Para la definición de políticas y sus niveles se desarrollaron cuatro grupos focales, compuestos por expertos, académicos y políticos. Como resultado de los encuentros se determinó que la renta básica y servicio gratuito a personas en situación de exclusión severa (PROGRAMA 1), y la subvención a empresas de inclusión social que emplean y forman para el empleo a grupos en riesgo de exclusión (PROGRAMA 2) son medidas vigentes en la región de estudio, y con un determinado impacto social, mientras que la compra pública responsable (PROGRAMA 3) y los programas de sensibilización (PROGRAMA 4) aún no forman parte de la agenda de trabajo en materia de política social. Así, el trabajo con expertos permitió definir cuatro tipos de programas, cuyo desarrollo o implantación son técnicamente viables en el territorio. Estas reuniones también sirvieron para establecer los límites óptimos de intervención de dichas políticas (Cuadro 2). 
Cuadro 2. Situación actual y propuesta de desarrollo de las políticas de inclusión social en Canarias.

\begin{tabular}{|c|c|c|c|c|}
\hline Programa & $\begin{array}{l}\text { Situación } \\
\text { Actual }\end{array}$ & $\begin{array}{l}\text { Nivel } \\
\text { Bajo }\end{array}$ & $\begin{array}{l}\text { Nivel } \\
\text { Medio }\end{array}$ & $\begin{array}{l}\text { Nivel } \\
\text { Alto }\end{array}$ \\
\hline $\begin{array}{l}\text { Renta básica y } \\
\text { servicios gratuitos }\end{array}$ & $\begin{array}{l}\cdot 130.000 \text { en } \\
\text { exclusión severa } \\
\cdot 7.000 \text { reciben } \\
\text { prestación de } \\
\text { inserción }(5,5 \%)\end{array}$ & $\begin{array}{l}\cdot \mathbf{2 5} \% \text { recibiría } \\
\text { prestación. } \\
\cdot \mathbf{3 2 . 5 0 0} \text { personas }\end{array}$ & $\begin{array}{l}\cdot 50 \% \text { recibiría } \\
\text { prestación. } \\
\cdot 65.000 \\
\text { personas }\end{array}$ & $\begin{array}{l}\text {-75\% recibiría } \\
\text { prestación. } \\
\cdot \mathbf{9 7 . 5 0 0} \\
\text { personas }\end{array}$ \\
\hline $\begin{array}{l}\text { Subvención a } \\
\text { empresas que } \\
\text { emplean y forman }\end{array}$ & $\begin{array}{l}\text { Unos } 1.600 \text { empleos } \\
\text { subvencionados entre } \\
\text { E.I. y C.E.E. }\end{array}$ & $\begin{array}{l}\text { Creación de } \\
\mathbf{1 0 . 0 0 0} \text { empleos } \\
\text { subvencionados } \\
\text { más }\end{array}$ & & $\begin{array}{l}\text { Creación de } \\
\mathbf{2 0 . 0 0 0} \\
\text { empleos } \\
\text { subvencionados } \\
\text { más }\end{array}$ \\
\hline $\begin{array}{l}\text { Plan de compra } \\
\text { público } \\
\text { responsable }\end{array}$ & $\begin{array}{l}\text { Actualmente no hay } \\
\text { Plan de Compra } \\
\text { Pública Responsable }\end{array}$ & $\begin{array}{l}\text { Hasta un límite del } \\
\mathbf{5 \%} \text { de todas las } \\
\text { compras de las } \\
\text { Administraciones }\end{array}$ & & $\begin{array}{l}\text { Hasta un límite } \\
\text { del } \mathbf{1 0 \%} \text { de } \\
\text { todas las } \\
\text { compras de las } \\
\text { Administraciones }\end{array}$ \\
\hline $\begin{array}{l}\text { Sensibilizar a la } \\
\text { sociedad en } \\
\text { consumo } \\
\text { solidario }\end{array}$ & $\begin{array}{l}\text { Actualmente no hay } \\
\text { Plan de } \\
\text { Sensibilización de } \\
\text { Consumo Solidario }\end{array}$ & $\begin{array}{c}\text { Informar al } \mathbf{2 5 \%} \\
\text { de la población } \\
\text { canaria }\end{array}$ & $\begin{array}{c}\text { Informar al } \mathbf{5 0} \\
\text { \% de la } \\
\text { población } \\
\text { canaria }\end{array}$ & $\begin{array}{l}\text { Informar al } \\
\mathbf{7 5} \% \text { de la } \\
\text { población } \\
\text { canaria }\end{array}$ \\
\hline
\end{tabular}

Fuente: Elaboración propia.

Usando como base estas cuatro alternativas de programas y sus dimensiones, se generaron fichas de casos hipotéticos, en formato tarjetas, con varias combinaciones de programas de inserción y la cantidad de dinero que el individuo tendría que pagar para su financiación. En cada tarjeta, el encuestado debía elegir la combinación preferida, considerando que cada tarjeta contenía las únicas opciones posibles.

El valor monetario de la disposición a pagar (DAP) significaba la cuantía de una hipotética contribución especial cada año (un impuesto por ejemplo), que el individuo debería estar dispuesto a asumir con el fin de apoyar estos programas de inserción social, y que en caso de que se lleven a cabo, todos los ciudadanos tendrían que pagar también para financiarlos.

Matemáticamente, la probabilidad de elección de los individuos de la política preferida se puede expresar en términos de distribución logística (Mcfadden,1973), conocido como modelo logit condicional, tal y como se expresa en la ecuación 1.1: 


$$
P\left(U_{i g}>U_{i h}, \forall h \neq g\right)=\frac{\exp \left(\mu \mathrm{V}_{\mathrm{ig}}\right)}{\sum_{\mathrm{j}} \exp \left(\mu \mathrm{V}_{\mathrm{ij}}\right)}
$$

En este tipo de modelo, la función de utilidad de cada individuo de elegir una alternativa i (U) se descompone en dos partes principales: un elemento determinístico-lineal (V), y uno estocástico (e), que representa factores determinantes no directamente observables. Entonces, la probabilidad de que un individuo prefiera la opción g puede expresarse como la probabilidad de que la utilidad asociada a elegir g exceda a la utilidad que le produce cualquiera de las otras opciones.

En este caso, $\mu$ corresponde con el parámetro de escala, que es inversamente proporcional a la desviación estándar del error. Este parámetro por lo general se asume igual a 1. El modelo se estima mediante un procedimiento convencional de máxima verosimilitud.

Los parámetros a estimar significan una medida monetaria de bienestar social para los individuos por elegir la opción preferida, que puede establecerse para cada atributo del modelo, con la fórmula 1.2:

$$
\text { [1.2] Monetary value }=\frac{\ln \left\{\frac{\sum_{\mathrm{i}} \exp \left(\mathrm{V}_{\mathrm{i}}^{1}\right)}{\sum_{\mathrm{i}} \exp \left(\mathrm{V}_{\mathrm{i}}^{0}\right)}\right\}}{b_{c}}
$$

Las combinaciones de programas y niveles se obtuvieron a partir del método eficiente bayesiano usando el programa Ngene. Con un total de quince niveles pertenecientes a los cuatro programas, se obtuvieron 12 combinaciones de programas. Cada tarjeta de elección presentaba 2 combinaciones y una tercera que era fija, correspondiente a la situación actual. Los precios de las tarjetas se consideran otro atributo del modelo, y fueron establecidos en torno a 20,40, 80, y 120 euros al año y asignados aleatoriamente a cada combinación. La figura 3 muestra un ejemplo de una de las tarjetas. 
Cuadro 3. Ejemplo de tarjeta utilizada en el análisis de las preferencias y la DAP por políticas de inclusión social.

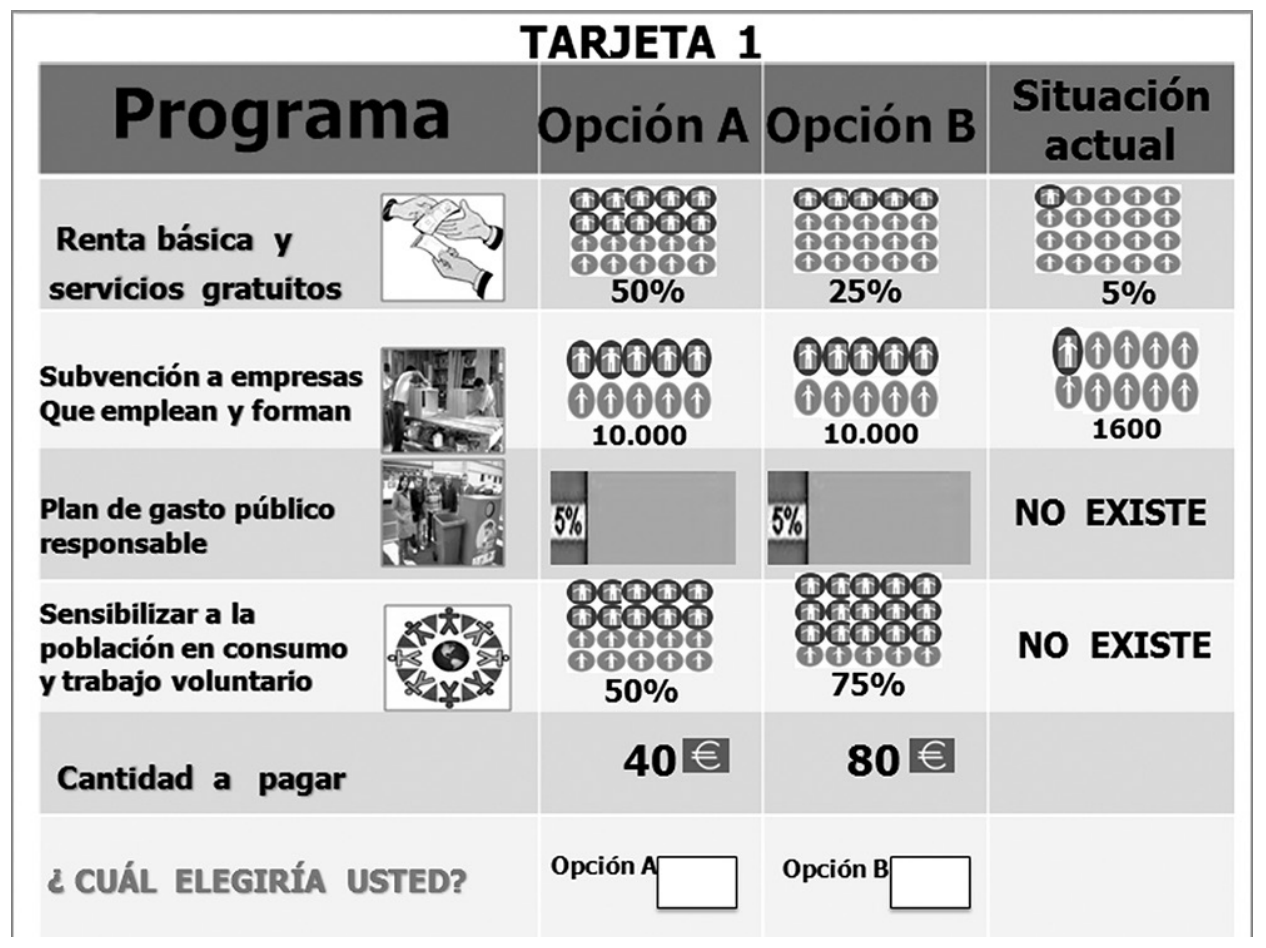

Fuente: Elaboración propia.

\subsection{Cuestionario}

La herramienta de investigación utilizada fue el cuestionario, el que se estructuró en cuatro bloques de preguntas abiertas, cerradas y de elección múltiple, a saber; i) Características socio demográficas del encuestado, ii) Conocimiento y evaluación de las causas y características de la exclusión social, iii) Satisfacción percibida por la implementación de medidas de inclusión social y iv) Valoración económica de programas con contenido solidario (tarjetas).

La encuesta recababa información sobre el nivel de conocimiento, la evaluación y la opinión del tejido social del archipiélago con respecto a las diferentes situaciones de exclusión social y las causas que la originan. A tal efecto, y con el fin de facilitar la comprensión por parte de la sociedad, se presentaron preguntas de valoración sobre las principales situaciones de exclusión, o de riesgo de exclusión, y los colectivos sociales afectados, tal y como se han definido en el estudio, a saber: 
- Los parados de larga duración sin cobertura de desempleo suficiente, y sin otros medios para la cobertura de las necesidades fundamentales personales y familiares.

- Las personas mayores sin recursos propios o transferidos para satisfacción de sus necesidades fundamentales.

- Las personas con alguna discapacidad física o mental de la cual se derive merma de capacidades para la satisfacción autónoma de sus necesidades, en el marco socio institucional en el que se desenvuelven.

- Mujeres cabeza de familias monoparentales con hijos a cargo y sin recursos suficientes para la cobertura de las necesidades propias y familiares fundamentales.

- Inmigrantes con derechos reducidos, que deben enfrentar dificultades añadidas para entrar en el mercado de trabajo y/o situaciones de sobreexplotación.

La encuesta fue administrada a 1250 personas, hombres y mujeres, residentes en Canarias, con una edad entre 33 y 45 años. Este se considera el rango de edad con mayor índice de inserción laboral activa en la región, y por tanto de mayores ingresos. Antes de realizar el trabajo de campo se realizó otro grupo focal con 10 residentes canarios, para validar las preguntas del cuestionario y corregir aquellos aspectos que podrían generar confusión en el encuestado.

Una de las principales conclusiones de esta fase de validación del cuestionario fue la necesidad de repartir las 12 combinaciones (tarjetas) de programas en diferentes cuestionarios, de manera que cada individuo solo respondiera a dos tarjetas (cuatro combinaciones). De este modo se optó por preparar tres modelos diferentes de cuestionarios, siendo las tarjetas la única preguntaba que variaba.

Para la encuestación, se utilizó una empresa especializada en estudio de mercado. El trabajo de campo abarcó diez meses culminando en enero 2018. Los diferentes modelos de cuestionarios se repartían de manera aleatoria entre los encuestados, hasta llegar a la misma cantidad de encuestas para cada modelo.

Se utilizó el paquete estadístico STATA para el tratamiento de los datos. El análisis descriptivo se utilizó para identificar el perfil de los encuestados, su nivel de conocimiento sobre las causas de exclusión social, los tipos de exclusión social que existen y su satisfacción con respecto a las medidas que combaten estas situaciones. Por su parte la regresión ASC logit permitió explicar en qué medida los factores demográficos o socio-psicológicos de los individuos explican una mayor disposición a pagar (DAP). Además, se obtuvo un valor medio de la DAP por cada PROGRAMA, a partir del análisis de todas las respuestas.

\section{Resultados}

En cuanto a las características de la muestra, ha de destacarse que el 30,7\% de los encuestados se encontraban desempleados en el momento de responder el cuestionario, representando el mayor porcentaje, seguido por los trabajadores por cuenta ajena. La mayoría de los encuestados es hombre (65,8\%), con un nivel formativo equivalente a Bachiller. Más del 30\% de los en- 
cuestados declaró que tenía ingresos mensuales por debajo de 400 euros, que solo le permite cubrir las necesidades básicas (tabla 2).

Tabla 2. Características sociodemográficas de los encuestados.

\begin{tabular}{|c|c|c|}
\hline Variable & Categoría & Frecuencia \\
\hline \multirow[t]{2}{*}{ Sexo } & Hombre & 65,9 \\
\hline & Mujer & 34,1 \\
\hline \multirow[t]{2}{*}{ Pareja } & $\mathrm{Si}$ & 57,1 \\
\hline & No & 42,9 \\
\hline \multirow[t]{4}{*}{ Formación } & Bachiller o Ciclo Formativo & 42,0 \\
\hline & Licenciatura & 18,5 \\
\hline & Enseñanza secundaria & 15,1 \\
\hline & Diplomatura & 15,1 \\
\hline \multirow[t]{4}{*}{ Ocupación } & Desempleado & 30,7 \\
\hline & Trabajador por cuenta ajena & 30,2 \\
\hline & Estudiante & 11,7 \\
\hline & Autónomo & 8,8 \\
\hline \multirow[t]{4}{*}{ Situación económica } & Solo puedo permitirme lo básico & 31,2 \\
\hline & Puedo permitirme algunos lujos & 29,8 \\
\hline & No puedo permitirme lujos & 23,4 \\
\hline & Tengo lo suficiente para vivir & 10,7 \\
\hline \multirow[t]{4}{*}{ Nivel de ingresos } & Menos de $400 €$ & 34,1 \\
\hline & $401-600 €$ & 16,1 \\
\hline & $1001-1500 €$ & 16,1 \\
\hline & $801-1000 €$ & 12,7 \\
\hline
\end{tabular}

Fuente: Elaboración propia. 


\subsection{Nivel de conocimiento sobre la exclusión social y satisfacción por las medidas para erradicarla}

La primera valoración que se obtiene de los encuestados se refiere a su nivel de conocimiento sobre las causas que originan una situación de exclusión social. Para ello, debían valorar ocho aspectos en base a una escala Likert de cinco puntos (siendo $1=$ Nada de acuerdo y $5=$ Completamente de acuerdo). Según la mayor parte de los encuestados, una situación de exclusión viene a ser provocada fundamentalmente por haber perdido un empleo y no recuperarlo, y por no tener una jubilación suficiente. A modo general, tomando en cuenta que las valoraciones medias están por encima de 3,2, se puede afirmar que los encuestados han reconocido a todas las causas como factores de exclusión social, aunque no estén completamente seguros de ello. (Tabla 3).

Tabla 3. Causas principales que conllevan a una situación de exclusión social.

\begin{tabular}{lr} 
Aspectos & Media \\
\hline Tiene adicción a drogas, juego, alcohol, etc. & 3,50 \\
\hline No ha tomado suficiente responsabilidad de su vida & 3,20 \\
\hline No tienen una pensión de jubilación o que esta fuera insuficiente & 3,93 \\
\hline No están cubiertos por la prestación de desempleo & 3,57 \\
\hline Han perdido el empleo y no han vuelto a encontrar & 4,00 \\
\hline Nunca han tenido un empleo & 3,25 \\
\hline Viven en el seno de una familia desestructurada & 3,43 \\
\hline No han recibido la formación escolar adecuada & 3,62
\end{tabular}

1= Nada de acuerdo; 5= Completamente de acuerdo

Fuente: Elaboración propia.

Los encuestados también debían identificar los colectivos que a su criterio eran más propensos a encontrarse en una situación de exclusión social. La sociedad identifica a las personas mayores sin recursos como el grupo más vulnerable a una situación de exclusión, seguido por los desempleados de larga duración. Los autónomos y pequeños agricultores con bajos ingresos es el grupo que como media se considera menos expuesto a una situación de precariedad (Tabla 4). Al igual que en el caso anterior, el hecho de obtener valoraciones medias por debajo del punto 4 , indica que es poco frecuente que los encuestados hayan respondido con total 
seguridad a la pregunta. Este es un resultado a tener en cuenta toda vez que la identificación de estos colectivos como vulnerables por parte de la sociedad es un paso fundamental para alentar su apoyo, lo que lógicamente también dependerá de la efectividad de las estrategias de sensibilización.

\section{Tabla 4. Grupos sociales con alto riesgo de exclusión social.}

\begin{tabular}{lr} 
Aspectos & Media \\
\hline Pequeños agricultores y autónomos de bajos ingresos & 3,27 \\
\hline Inmigrantes con derechos reducidos & 3,40 \\
\hline Madres en familia monoparentales & 3,37 \\
\hline Personas con adicción a drogas, alcohol, etc. & 3,62 \\
\hline Personas con discapacidad física, psíquica & 3,82 \\
\hline Personas mayores sin recursos & 3,98 \\
\hline Desempleados de larga duración & 3,82 \\
\hline
\end{tabular}

$1=$ no pertenecen de ningún modo a 5= pertenecen con total seguridad

Fuente: Elaboración propia.

Por su parte, los encuestados indicaron el grado de satisfacción (_sat) que les produce algunas medidas destinadas a personas en situación de exclusión. Proporcionar la formación profesional y experiencia laboral adecuada a las personas es la medida que más satisfacción produce entre los encuestados, seguido de la garantía de renta básica. En general, todas las medidas generan satisfacción entre los individuos (Tabla 5). Estos resultados fueron incluidos en el modelo, toda vez que permitiría identificar si mayores niveles de satisfacción por la existencia de medidas determinan una mayor disposición a pagar de los individuos por promover su implementación. 


\section{Tabla 5. Satisfacción percibida por las medidas en favor de la inclusión social (_sat).}

\begin{tabular}{lc} 
Aspectos & Media \\
\hline Ayudar a empresas que contratan personas en situación de exclusión & 4,03 \\
\hline $\begin{array}{l}\text { Promocionar la compra de bienes y servicios producidos por empresas que } \\
\text { promueven la inclusión }\end{array}$ & 3,80 \\
\hline Apoyar la creación de empresas con financiación y asistencia técnica & 3,91 \\
\hline Proporcionar formación profesional y experiencia laboral & 4,21 \\
\hline Proporcionar acceso gratuito a servicios fundamentales & 4,10 \\
\hline Garantizar la renta mínima a familias & 4,16 \\
\hline
\end{tabular}

1= Nada satisfecho; 5= Muy satisfecho

Fuente: Elaboración propia.

\subsection{Resultados del modelo}

Para valorar las preferencias sobre políticas, los encuestados debían elegir una combinación de medidas y la cantidad de dinero que podrían estar dispuestos a pagar para su financiación. Los valores se corresponden con una cantidad anual (impuesto) que el encuestado estaría dispuesto a asumir para garantizar la puesta en marcha de un programa determinado, y sabiendo que el conjunto de la población de Canarias también estaría obligado al pago de esta contribución. Tal y como se explicó en el apartado metodológico existían 12 combinaciones de políticas que se distribuyen en tarjetas de elección, asignando aleatoriamente un precio a cada combinación.

Del conjunto de respuestas se puede extraer que existe una alta sensibilidad al precio, pues siempre los encuestados eligieron la combinación que significaba pagar menos. En otras palabras, el 68\% de los encuestados siempre eligió la combinación de políticas que tenía asignado el menor precio, o sea 20 euros, siendo este precio asignado en las tarjetas a varias medidas, y no una sola política. En cuanto a políticas, las preferencias se inclinan hacia la transferencia de fondos para sustentar renta básica a las familias que lo necesitan, seguido de la subvención a empresas. En resumen, los encuestados están dispuestos a pagar más por aquellos programas que pagan directamente a las personas en riesgo de pobreza o exclusión (PROGRAMA 1 = $60,30 €)$, que por aquellos programas que fomentan el consumo de bienes y servicios por parte del resto de la sociedad hacia las empresas solidarias o que se dedican a contratar a personas en este tipo de situaciones (PROGRAMA 4=38,00€). En resumen, los encuestados prefieren como media las políticas públicas que directamente provean rentas o servicios gratuitos a colectivos en situación de exclusión social (Tabla 6). 
Tabla 6. Disposición a pagar por los programas de exclusión social.

\begin{tabular}{|c|c|c|c|}
\hline Variable & Coef. & Std. Err. & DAP \\
\hline Renta básica (PROGRAMA 1) & $0.49^{* \cdots * 2}$ & 0,04 & $60,30 €$ \\
\hline Subvención a empresas (PROGRAMA 2) & $0.31^{\text {k* }}$ & 0,03 & $52,30 €$ \\
\hline Plan de gasto público responsable (PROGRAMA 3) & $0.34^{\text {हैंसे }}$ & 0,04 & $42,00 €$ \\
\hline Sensibilizar para el consumo (PROGRAMA 4) & $0.29^{\text {*से }}$ & 0,03 & $38,00 €$ \\
\hline hombre_sexo & $-0.14^{* \cdots *}$ & 0,05 & \\
\hline no_pareja & $0,47^{\text {*k* }}$ & 0,02 & \\
\hline formación & $-0,74$ & 0,20 & \\
\hline cuenta ajena_ocupación & 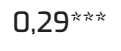 & 0,01 & \\
\hline autónomo_ocupación & 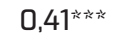 & 0,01 & \\
\hline nivel de ingresos & $-0,12$ & 0,15 & \\
\hline situación económica & -0.17 & 0,21 & \\
\hline ayudar a empresas_sat & 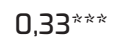 & 0,01 & \\
\hline promocionar compra de bienes y servicios_sat & $0,35^{\text {补衣 }}$ & 0,01 & \\
\hline proporcionar formación profesional_sat & $0,56^{* \cdots * 2 k}$ & 0,00 & \\
\hline _cons & 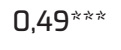 & 0,00 & \\
\hline $\mathrm{N}$ & 1250 & & \\
\hline $\mathrm{Chi}^{2}$ & 962,96 & 0,00 & \\
\hline
\end{tabular}

Fuente: Elaboración propia.

A nivel socio-demográfico las variables_sexo,_pareja y_ocupación fueron las únicas significativas. Los datos indican que los hombres sin pareja tienen una mayor probabilidad de pagar más en apoyo a la inclusión social. Asimismo, como es de esperar, contar con un empleo o con una actividad económica propia también aumenta la probabilidad de elegir la combinación de políticas con mayor precio, con respecto a la categoría de referencia (estar desempleado). Por último, los datos revelan que los encuestados con mayor DAP son los que han declarado una mayor satisfacción con las medidas relacionadas con el apoyo a las empresas, la promoción de bienes y servicios y la formación profesional. 


\section{Discusión y conclusiones}

Las personas en situación de pobreza y exclusión social son aquellas que están privadas de los medios necesarios para cubrir sus necesidades básicas (alimentación, educación, vivienda, etc...) y marginadas de las oportunidades para hacerlo posible. Pueden haber llegado a esta situación por permanecer largo tiempo en desempleo o haber perdido su pequeño negocio, haber tenido problemas con alcohol y otras drogas, tener algún tipo de discapacidad física 0 psíquica, ser madres solteras con hijos a cargo, personas mayores con escasos ingresos y cuidados, inmigrantes irregulares, etc.

Para apoyar a estos colectivos a salir de la situación de pobreza y exclusión, pueden llevarse a cabo diversos programas públicos y otras iniciativas provenientes del Estado, organizaciones sin fines de lucro y grupos que defienden la economía social solidaria. Todos estos programas y acciones tienen costes, y cuanto mayor sea la financiación de esos programas, más personas podrán ser atendidas y beneficiadas. Por este motivo, la lucha por la inclusión social es también responsabilidad de toda la sociedad, que tiene herramientas socio-económicas fundamentales para impulsar, apoyar y transformar estos procesos. Por ello, es fundamental que la sociedad esté informada y sensibilizada con el problema, y sea capaz de distinguir las políticas más eficientes y efectivas en cuanto al uso y destino de recursos financieros se refiere.

Los resultados de este estudio suponen un importante avance en este sentido, ya que ha permitido entender en qué áreas radican las principales debilidades de las políticas actuales en Canarias que buscan sensibilizar a la población sobre las formas más eficientes de apoyo a la inclusión social. Si bien los resultados podrían variar a escala nacional, aquí se muestra una guía metodológica de potencial aplicación al caso español.

Se ha encontrado que existe una alta preferencia en la sociedad (mayor DAP) por las políticas públicas que directamente provean rentas o servicios gratuitos, y menos interés por otras alternativas de promoción del gasto y el consumo responsable, que en definitiva no es más que la promoción de la Economía Social y Solidaria.

Promover el gasto o consumo responsable no es más que la concesión de subvenciones a la producción, destinada a las empresas que contratan determinados colectivos en riesgo de exclusión. Estas subvenciones a la producción pueden ser también excepciones de costes en elementos concretos del inmovilizado, como edificios, maquinaria, mobiliario, etc. En todos los casos, se trata de abaratar sus costes favoreciendo de este modo que estas empresas social-solidarias puedan competir con las empresas convencionales. En este contexto, es preciso que la sociedad entienda la utilidad que tiene destinar la financiación a este tejido productivo que produce exactamente los mismos beneficios, cualquiera que sea la política de inclusión social, pero con un valor añadido sobre la renta básica o directa, que es la de la inserción laboral.

Se trata entonces de reorientar los objetivos de los programas de sensibilización ciudadana, y por supuesto de las políticas públicas que persiguen acompañar su desarrollo y consolidación. Esto es, aceptar que el peso de las subvenciones directa va a disminuir y complementariamente estimular la demanda pública y privada de los bienes y servicios producidos por las entidades de economía solidaria (consumo responsable). En otras latitudes, incluyendo 
archipiélagos de la Macaronesia, se han desarrollado notables esfuerzos en el ámbito de la compra pública responsable, que han servido para generar una demanda suficiente y garantizar mínimos operativos a las empresas solidarias.

El fundamento de esas iniciativas se basa en la evolución de las preferencias sociales con respecto a los ejes e instrumentos de promoción de la economía solidaria. En efecto, el recurso a las subvenciones es cada vez más visto como un enfoque ineficiente que no estimula la innovación y puede llegar a encubrir comportamientos alejados del ideario de la economía solidaria. Por otra parte, la progresión de la economía solidaria parece poco probable que ocurra si no cambian también los valores que animan el comportamiento del conjunto de las organizaciones económicas y de la ciudadanía en tanto que actores económicos (consumidores, inversores, ahorradores, etc.).

En materia de políticas públicas Canarias debe esforzarse aún más para visibilizar y sensibilizar a la sociedad respecto a la pobreza y la exclusión social, motivando su participación en la compra de bienes y servicios a las EES (proporcionar trabajo voluntario, asistir a movilizaciones o actividades, y/o contribuir en especie al sector), ya que de esto depende el futuro de la Economía Solidaria. Esto requiere reorientar las políticas hacia el estímulo de la demanda, aceptando que el peso de las subvenciones va a disminuir. Por su parte, la Compra Pública responsable es una alternativa eficiente ya que permite multiplicar el impacto de las acciones públicas en favor de este sector.

Por último, en el contexto de economías con fuerte especialización en el turismo, como es el caso de Canarias, el enfoque de vincular la fortaleza de las entidades de economía solidaria con la solidez de sus vínculos con el conjunto del tejido económico y social, cobra todo su sentido. En efecto, el objeto de consumo de la actividad turística son experiencias personales ligada a la visita de un determinado entorno ambiental y sociocultural. A partir de ahí, la literatura publicada constata que es creciente la preferencia de la ciudadanía en tiempo de ocio (turistas) por vivir experiencias que generen una huella ambiental y social positiva. En consecuencia, el establecimiento de un vínculo positivo, mutuamente beneficioso, entre empresas turísticas y entidades de economía solidaria, parece no sólo viable, sino deseable desde el punto de vista de la sostenibilidad de ambas.

Contribución de cada autor/a: Conceptualización, M.M.G.H.; trabajo de campo y tratamiento de los datos, Y.E.L.-G; Análisis, M.M.G.H., Y.E.L.; método, M.M.G.H., Y.E.L; software, Y.E.L.; supervisión, M.M.G.H.; visualización, Y.E.L.G. borrador, Y.E.L., revision y edición Y.E.L.-G.,y M-M.G.H.

Financiación: Estudio financiado por el Programa Interreg MAC a través del proyecto ECOS- Sustentabilidad integrada de las empresas y organizaciones de Economía Solidaria (MAC/3/C128). 


\section{Bibliografía}

AGULNIK, P. (2002): Understanding social exclusion, 0xford: University Press on Demand.

ANDRÉ MANCE, E. (2007): Solidarity Economics, New York: Mimeo.

AROPE (2020): El estado de la pobreza. Seguimiento de indicadores de pobreza y exclusión social en España 2008-2019. 10 Informe, 2020. Disponible en: https://www.eapn.es/estadodepobreza/ARCHIVO/documentos/Informe_AROPE_2020_Resumen_Ejecutivo_73kN5F2.pdf (fecha consulta 10/05/2021).

BONO, E. (2012): "El decrecimiento sostenible, crisis ecológico-económica, desigualdad y economía social", CIRIEC-España, Revista de Economía Pública, Social y Cooperativa, 76, 181-196.

BORZAGA, C., SALVATORI, G. \& BODINI, R. (2019): "Social and solidarity economy and the future of work", Journal of Entrepreneurship and Innovation in Emerging Economies, 5(1), 37-57. DOI: $10.1177 / 2393957518815300$

CASASSAS, D. (2013): “Comercio y emancipación social en el republicanismo de Adam Smith: Una lectura contemporánea”, Mediterráneo Económico, 23, 31-49.

DECERF, B. (2021): "Combining absolute and relative poverty: income poverty measurement with two poverty lines", Social Choice and Welfare, 56(2), 325-362.

GOTTLIEB, A. (2017): "Incarceration and relative poverty in cross-national perspective: The moderating roles of female employment and the welfare state", Social Service Review, 91(2), 293-318. DOI: $10.1086 / 692357$

CASTRO, R., SANTERO, R., MARTíNEZ, M.I. \& GUILLÓ, N. (2013): “Impacto socioeconómico de las empresas de Economía Social en España. Una valoración cuantitativa de sus efectos sobre la cohesión social", CIRIEC-España, Revista de Economía Pública, Social y Cooperativa, 79, 35-58.

DELGADO, O.B. \& MADELEINE, R. (2001): “Economía social y economía solidaria: intento de definición", Cayapa, Revista venezolana de economía social, 1(1), 0.

FELBER, C. (2012): La economía del bien común, Barcelona: Deusto 5aํ Edición.

GONZÁLEZ HERNÁNDEZ, M.M., LAM GONZÁLEZ, Y.E., AMARO, ROGERIO. R. \& GIMÉNEZ RODRÍGUEZ, A. (2013): “Conocer la Economía Solidaria”. En: Economía Solidaria. Aportaciones del proyecto ECOS, 9-65m, Las Palmas de Gran Canaria: Radio Ecca, Fundación canaria 12/2013.

HODGSON GARABOTE, I. (2019): La Evolución del mercado laboral español, con especial referencia a la Comunidad Autónoma de Canarias.

HORRACH, P. \& SALVÀ, A. S. (2011): "La actitud de las empresas de economía solidaria frente a la divulgación de información sobre sostenibilidad desde el prisma de la teoría de los stakeholders o grupos de interés", Revista de contabilidad 14, 267-297. D0I: 10.1016/S11384891(11)70035-8. 
IBAÑEZ, M., FORMICHELLA, M. \& EZEQUIEL, L. (2020): "Exclusión social: explorando la dimensión educativa en Argentina", Problemas del Desarrollo. Revista Latinoamericana de Economía, 51 (200), 103-128, https://doi.org/10.22201/iiec.20078951e.2020.200.68518.

LEE, S. (2020): "Role of social and solidarity economy in localizing the sustainable development goals", International Journal of Sustainable Development \& World Ecology, 27(1), 65-71. DOI:10.1080/13504509.2019.1670274.

LITTLEWOOD, P. \& HERKOMMER, S. (2017): "Identifying social exclusion: Some problems of meaning", Social Exclusion in Europe, Routledge, 1-22.

LLANO-ORTIZ, J.C. (2016): El estado de la pobreza. Seguimiento del indicador de riesgo de pobreza y exclusión social en España 2009-2015, Madrid: EAPN.

MCFADDEN, D. (1973): Conditional logit analysis of qualitative choice behavior.

NAVARRO, C.L. \& MUÑOZ ARCE, G. (2016): "Revisitando el concepto de exclusión social: su relevancia para las políticas contra la pobreza en América Latina", Revista del CLAD Reforma y Democracia, 65, 39-68. D0I: 10.4025/dialogos.v21i2.13.

NOLAN, B. \& WHELAN, C.T. (1996): Resources, deprivation, and poverty. OUP Catalogue.

PALOMINO, J.C., RODRIGUEZ, J.G. \& SEBASTIAN, R. (2021): "The COVID-19 shock on the labour market: Poverty and inequality effects across Spanish regions", Available at SSRN 3775091. DOI: 10.2139/ssrn.3775091

PEDREÑO, M.H. (2010): "El estudio de la pobreza y la exclusión social. Aproximación cuantitativa y cualitativa”, Revista interuniversitaria de formación del profesorado, 24 (3), 25-46.

PÉREZ NÚÑEZ, U.V. (2005): Políticas públicas contra la exclusión social en gobiernos locales: el caso de Sant Boi de Llobregat, tesina.

SALVÀ, A.S. \& ROSSELLÓ, P.H. (2013): “Enfoque de la responsabilidad social y la transparencia en empresas de economía solidaria”, CIRIEC-España, Revista de Economía Pública, Social y Cooperativa, 77, 31-57.

SCHILLER, B.R. (1989): The economics of poverty and discrimination, Englewood Cliffs, NJ: Prentice Hall.

SJÅSTAD, H., ZHANG, M., MASVIE, A.E. \& BAUMEISTER, R. (2021): "Social exclusion reduces happiness by creating expectations of future rejection", Self and Identity, 20(1), 116-125. DOI: 10.1080/15298868.2020.1779119.

TORRESANO, D. et al. (2015): Economía solidaria: experiencias y conceptos, Ecuador: Mangraf. Disponible en: https://base.socioeco.org/docs/2705a873-b256-4f6a-8dfd-59370b09f0d4.pdf 